\title{
Xenograft models for undifferentiated pleomorphic sarcoma not otherwise specified are essential for preclinical testing of therapeutic agents
}

\author{
MARC BECKER ${ }^{1,2 *}$, CLAUDINE GRAF $^{3 *}$, MARCUS TONAK $^{2,4}$, MARKUS P. RADSAK $^{5}$, \\ TOBIAS BOPP ${ }^{5}$, ROBERT BALS ${ }^{6}$, RAINER M. BOHLE ${ }^{1}$, MATTHIAS THEOBALD ${ }^{3}$, \\ POL-MARIA ROMMENS ${ }^{2}$, DIRK PROSCHEK $^{2 * *}$ and THOMAS C. WEHLER ${ }^{3 * *}$ \\ ${ }^{1}$ Institute of Pathology, Saarland University Medical Center, Homburg, Saarland D-66421; \\ ${ }^{2}$ Interdisciplinary Sarcoma Working Group, Center of Orthopaedic and Trauma Surgery; \\ ${ }^{3}$ III Department of Internal Medicine, University Medical Center, Johannes Gutenberg University of Mainz, \\ Mainz, Rhineland-Palatinate D-55131; ${ }^{4}$ Department for Orthopaedic and Trauma Surgery, University Hospital Mannheim, \\ Mannheim, Baden-Württemberg D-68167; Institute for Immunology, University Medical Center, \\ Johannes Gutenberg University of Mainz, Mainz, Rhineland-Palatinate D-55131; \\ ${ }^{6}$ Department of Internal Medicine V - Pulmonology, Allergology and Respiratory Critical Care Medicine, \\ Saarland University Medical Center, Homburg, Saarland D-66421, Germany
}

Received April 27, 2015; Accepted December 11, 2015

DOI: $10.3892 / \mathrm{ol} .2016 .4784$

\begin{abstract}
Undifferentiated pleomorphic sarcoma not otherwise specified belongs to the heterogeneous group of soft tissue tumors. It is preferentially located in the upper and lower extremities of the body, and surgical resection remains the only curative treatment. Preclinical animal models are crucial to improve the development of novel chemotherapeutic agents for the treatment of undifferentiated pleomorphic sarcoma. However, this approach has been hampered by the lack of reproducible animal models. The present study established two xenograft animal models generated from stable non-clonal cell cultures, and investigated the difference in chemotherapeutic effects on tumor growth between undifferentiated pleomorphic sarcoma in vivo and in vitro. The cell cultures were generated from freshly isolated tumor tissues of two patients with undifferentiated pleomorphic sarcoma. For the in vivo analysis, these cells were injected subcutaneously into immunodeficient mice. The mice were monitored for tumor appearance and treated with the most common or innovative chemotherapeutic agents available to date. Furthermore, the same drugs were administered to in vitro cell
\end{abstract}

Correspondence to: Dr Thomas C. Wehler, III Department of Internal Medicine, University Medical Center, Johannes Gutenberg University of Mainz, Mainz, Rhineland-Palatinate D-55131, Germany E-mail: thomas.wehler@uks.eu

*Contributed equally; ${ }^{* *}$ Joint senior authorship

Key words: pleomorphic sarcoma, doxorubicin, pazopanib, HDAC inhibitor, xenograft, animal model cultures. The most effective tumor growth inhibition in vitro was observed with doxorubicin and the histone deacetylase inhibitor suberoylanilide hydroxamic acid (SAHA), also known as vorinostat. In the in vivo xenograft mouse model, the combination of doxorubicin and the tyrosine kinase inhibitor pazopanib induced a significant tumor reduction. By contrast, treatment with vorinostat did not reduce the tumor growth. Taken together, the results obtained from drug testing in vitro differed significantly from the in vivo results. Therefore, the novel and reproducible xenograft animal model established in the present study demonstrated that in vivo models are required to test potential chemotherapeutic agents for the treatment of undifferentiated pleomorphic sarcoma prior to clinical use, since animal models are more similar to humans, compared with in vitro cell cultures.

\section{Introduction}

Soft tissue sarcoma (STS) is a heterogeneous group of neoplasms arising from degenerated cells of mesenchymal origin (1). Currently, STS is differentiated into $>20$ distinct subtypes, which are classified according to their tissue of origin (2). The most common type of STS observed in adults is undifferentiated pleomorphic sarcoma not otherwise specified (NOS), which was previously known as malignant fibrous histiocytoma, and presents five histological subtypes (3-5). Currently, surgical resection remains the only method of curative treatment for undifferentiated pleomorphic sarcoma NOS, which often occurs at a high malignancy grade, possesses a high risk of metastasis, and exhibits resistance to radiotherapy and chemotherapy (6-8). Previous studies have demonstrated that additional radiation therapy improves local tumor control; however, chemotherapy remains palliative, since there are 
no effective chemotherapy drugs $(9,10)$. Due to the limited number of patients with specific subtypes of undifferentiated pleomorphic sarcoma NOS, clinical studies often present limitations, and the majority of the data available may not apply to certain subtypes. The application of an anthracycline-based chemotherapy, including doxorubicin alone or in combination with ifosfamide, is often the first-line treatment of undifferentiated pleomorphic sarcoma NOS, and there are no widely recognized second-line therapies available (11-13). Recently, improved second-line drugs have been developed, including histone deacetylase (HDAC) inhibitors, trabectedin and tyrosine kinase inhibitors such as pazopanib, which are more effective than those currently available (14-19). With these drugs, a progression-free survival time of 3-5 months may be achieved (14-19). However, the overall survival time is not increased. Therefore, to prolong the survival time of patients with undifferentiated pleomorphic sarcoma NOS, additional improvements are required.

Cell lines and animal models are powerful tools for the development of innovative therapeutics. In the past recent years, numerous cell lines derived from undifferentiated pleomorphic sarcoma have been generated and characterized (20-24). A number of these cell lines were injected subcutaneously into immunodeficient mice, and 4 weeks subsequent to injection measurable tumor tissue was formed $(20,21,24)$. However, investigations comparing the effect of therapeutical approaches in vivo and in vitro have not been performed thus far. In other studies using xenotransplantation models, original human tumor tissue was transplanted into immunodeficient mice (25-27). These animal models appear to be more similar to the tumors observed in humans, but hypoxia following tissue transplantation remains a problem (25-27).

In the present study, two stable cell cultures were generated from two patients with undifferentiated pleomorphic sarcoma. These cells were subcutaneously injected into immunodeficient mice, whereby remained tumorigenic. Following chemotherapeutic treatment of the tumor, clear differences were observed between the in vitro and in vivo models, which confirms that it is imperative to test innovative chemotherapeutics in appropriate animal models prior to clinical use.

\section{Materials and methods}

Animals. In total, 4 immunodeficient non-obese diabetic (NOD) severe combined immunodeficiency (SCID) gamma (NSG) mice (NOD.Cg-Prkde ${ }^{\text {scid }} \mathrm{Il} 2 \mathrm{rg}^{\mathrm{tm} l \mathrm{w}_{\mathrm{jl}}} / \mathrm{SzJ}$ ) (2 male and 2 female; 8 weeks-old) were purchased from the Jackson Laboratory (Sacramento, ME, USA), and bred in the animal facility at the Johannes Gutenberg University of Mainz (Mainz, Germany). The mice were bred, maintained and manipulated under specific pathogen-free conditions. All the food, water and litter were sterilized prior to use. The temperature and humidity were controlled at $20-24^{\circ} \mathrm{C}$ and $45-65 \%$, respectively. Daily light cycles consisted of $12 \mathrm{~h}$ light and dark cycles. The cages were fully cleaned once or twice per week. Mice that were 6-8 weeks-old were used for subcutaneous injections. All animal procedures were conducted in accordance with the Institutional Guidelines of the Johannes Gutenberg University of Mainz, and approved by the responsible national authority (National Investigation
Office Rheinland-Pfalz; Koblenz, Germany; approval no. 23 177-07/G 13-1-027).

Isolation of sarcoma samples and cell culture. Stable oligoclonal cell cultures, termed MZ-UPS-1 and MZ-UPS-2, were generated from freshly isolated tumor tissue from two patients diagnosed with undifferentiated pleomorphic sarcoma NOS. These two patients were treated in 2011 and 2012, respectively, at the University Medical Center in Mainz and had undergone surgeries where the local tumor tissue was removed. Immediately following resection, the tumor tissue was minced, placed onto 6-well plates (Sigma-Aldrich Chemie $\mathrm{GmbH}$, Taufkirchen, Germany) and cultivated at $37^{\circ} \mathrm{C}$ in a humidified atmosphere with $5 \% \mathrm{CO}_{2}$ with Gibco $^{\circledR}$ Dulbecco's modified Eagle's medium [Nutrient Mixture F-12 containing GlutaMAX ${ }^{\mathrm{TM}}$ Supplement (DMEM/F12; Thermo Fisher Scientific, Inc., Waltham, MA, USA)], $1 \%$ sodium pyruvate (100 mM; Thermo Fisher Scientific, Inc.), 10\% fetal calf serum (FCS; GE Healthcare Life Sciences, Chalfont, UK) and $1 \%$ penicillin-streptomycin (Invitrogen ${ }^{\mathrm{TM}}$; Thermo Fisher Scientific, Inc.). The cells were expanded to form a sub-confluent layer of adherent cells 2-4 weeks subsequent to initial seeding. Subsequently, the adherent tumor cells were digested using Accutase (GE Healthcare Life Sciences) and transferred into $175 \mathrm{~cm}^{2}$ cell culture flasks (Sigma-Aldrich Chemie $\mathrm{GmbH}$ ). Since the MZ-UPS-1 cells were a fast-growing culture, they were passaged and serially subcultured at a dilution of 1:3-1:5 every week. By contrast, the MZ-UPS-2 cells were passaged every two weeks and serially subcultured at a dilution of 1:2. The two undifferentiated pleomorphic cell cultures MZ-UPS-1 and MZ-UPS-2 were maintained in vitro for $\sim 30$ passages for $>1$ year.

The two patients provided written informed consent for biobanking. The present study was approved by the Ethics Committee of the University Medical Center of Johannes Gutenberg University of Mainz [Mainz, Germany; approval no. 837.250.13 (8935)].

Chemotherapeutic treatment in vitro and cell viability assay. MZ-UPS-1 and MZ-UPS-2 cells from the fourth passage were harvested, washed in phosphate-buffered saline (PBS; Thermo Fisher Scientific, Inc.) and resuspended in DMEM/F12-GlutaMAX ${ }^{\mathrm{TM}}$ Supplement with $10 \%$ FCS. One day prior to chemotherapeutic treatment, the cells were seeded onto 96 -well plates $\left(1 \times 10^{4}\right.$ cells $/ 100 \mu \mathrm{l}$ medium/well; Greiner Bio-One GmbH, Frickenhausen, Germany) to ensure adherence. On day 1, the supernatants were discarded, and a cell viability assay was conducted with alamarBlue ${ }^{\mathrm{TM}}$ Cell Viability Assay Reagent (Thermo Fisher Scientific, Inc.), according to the manufacturer's protocol. Briefly, alamarBlue $^{\mathrm{TM}}$ solution was diluted 1:10 in DMEM/F12-GlutaMAX ${ }^{\mathrm{TM}}$ Supplement, and $100 \mu \mathrm{l} /$ well was added to the cells, which were incubated for 1-2 $\mathrm{h}$. Subsequently, the supernatants were removed, and the absorbance was measured at $570 \mathrm{~nm}$ (reference wavelength, $600 \mathrm{~nm}$ ) in a multiplate spectophotometer (Sunrise $^{\mathrm{TM}}$; Tecan Group Ltd., Männedorf, Switzerland). In parallel assays, the plated tumor cells were cultured in $200 \mu \mathrm{l}$ DMEM/F12-GlutaMAX ${ }^{\text {TM }}$ Supplement in the presence of the appropriate concentrations of the chemotherapeutic agents for $1,2,4,7,10$ and 14 days. alamarBlue ${ }^{\mathrm{TM}}$ assays were performed 
Table I. Chemotherapeutic treatment regimen of xenograft mouse models of undifferentiated pleomorphic sarcoma.

\begin{tabular}{lccc}
\hline Chemotherapy & Body concentration, $\mathrm{mg} / \mathrm{kg}$ & Type of application & Application mode \\
\hline Doxorubicin & 6 & Intravenous & Weekly \\
Suberoylanilide hydroxamic acid & 50 & Intraperitoneal & Daily \\
Pazopanib & 100 & Oral & Daily \\
\hline
\end{tabular}

at each of the above time points to determine cell viability. At least three experiments were performed.

Xenotransplantation. Cultured MZ-UPS-1 and MZ-UPS-2 cells from the fourth passage were harvested by detachment with Accutase and washed twice in PBS. Live cells were counted using trypan blue staining (Sigma-Aldrich Chemie $\mathrm{GmbH}$ ) and a Neubauer counting chamber (Sigma-Aldrich Chemie $\mathrm{GmbH}$ ). In total, $1 \times 10^{6}$ cells were injected subcutaneously into the right flank of NSG mice. Tumor growth was verified 4 weeks later. The effectiveness of the xenograft transplant was $100 \%$ for the two undifferentiated pleomorphic sarcoma cell cultures MZ-UPS-1 and MZ-UPS-2.

Histology. Samples from the original tumors of the two patients and isolated xenografts from the mice were fixed in $4 \%$ phosphate-buffered formaldehyde solution (Roti ${ }^{\circledR}$-Histofix; Carl Roth $\mathrm{GmbH}+\mathrm{Co} . \mathrm{KG}$, Karlsruhe, Germany) and embedded in paraffin (Sigma-Aldrich Chemie $\mathrm{GmbH}$ ). The tissue sections $(5 \mu \mathrm{m})$ were subsequently deparaffinized using xylol (Sigma-Aldrich Chemie $\mathrm{GmbH}$ ) and a descending sequence of ethanol (100, 90 and $70 \%$ and distilled water), stained with hematoxylin and eosin (Sigma-Aldrich Chemie $\mathrm{GmbH}$ ) and viewed under a microscope (Axioskop 40; Zeiss GmbH, Jena, Germany).

Immunohistochemical staining were performed according to manufacturers's protocol on a Ventana BenchMark XT platform (Ventana Medical Systems, Inc., Tucson, AZ, USA). Following deparaffinization, antigen retrieval was performed using peroxidase and alkaline phosphatase blocking reagent (Dako, Glostrup, Denmark) for $10 \mathrm{~min}$ at $95-99^{\circ} \mathrm{C}$. The tissue sections were incubated with mouse anti-human monoclonal vimentin (for $32 \mathrm{~min}$; catalog no., M0725), mouse anti-human monoclonal actin (for $32 \mathrm{~min}$; catalog no., M0851) and rabbit monoclonal anti-human Ki-67 antibodies (for 36 min; catalog no., M7240) at room temperature. All primary antibodies were purchased from Dako. Following washing two times with reaction buffer (Ventana Medical Systems, Inc.), the slides were then incubated with anti-mouse secondary antibody (ultraView Universal Alkaline Phosphatase Red Detection Kit; Ventana Medical Systems, Inc.) for $30 \mathrm{~min}$ at room temperature and visualized according to manufacturer's instructions.

Animal treatment and tumor measurement. The size of the tumors in the mice were measured using a digital caliper 6-8 weeks subsequent to subcutaneous injection of $1 \times 10^{6}$ MZ-UPS-1 or MZ-UPS-2 cells. Tumor size was measured from caudal to cranial and dorsal to ventral, and the values were multiplied to calculate the tumor area. Chemotherapeutic agents were prepared prior to application.
Ready-to-use doxorubicin was purchased at a concentration of $2 \mathrm{mg} / \mathrm{ml}$ (Hexal AG, Holzkirchen, Germany), and suberoylanilide hydroxamic acid (SAHA; Sigma-Aldrich Chemie $\mathrm{GmbH}$ ) was dissolved in dimethyl sulfoxide (DMSO; Sigma-Aldrich Chemie $\mathrm{GmbH}$ ) at a concentration of $25 \mathrm{mg} / \mathrm{ml}$. Pazopanib was purchased as $400 \mathrm{mg}$ capsules Votrient ${ }^{\circledR}$ (GlaxoSmithKline, Brentford, UK). One Votrient ${ }^{\circledR}$ capsule was ground using a pestel and mortar, and dissolved in DMSO at a concentration of $30 \mathrm{mg} / \mathrm{ml}$ upon mixing overnight. The mice were weighed and treated as described in Table I. Tumor size was measured every 2 days.

Statistical analysis. Student's $t$ test was used to compare the mean values between two experimental groups where appropriate using GraphPad Prism version 6.0f (GraphPad Software, Inc., La Jolla, CA, USA). For non-Gaussian distributions, the Mann Whitney U test was used for the calculation of statistical significance. Gaussian distribution was analyzed with the Kolmogorov Smirnov test. Data are expressed as the mean \pm standard deviation. $\mathrm{P}<0.05$ was considered to indicate a statistically significant difference.

\section{Results}

Two undifferentiated pleomorphic sarcoma cell lines were established. Histology of the biopsies and resected tumors of the two patients provided a diagnosis of undifferentiated pleomorphic sarcoma NOS grade 3 , according to the FNCLCC classification (28). The tumors were located in the left axillary region or the gluteus maximus. The two primary tumor tissues histologically exhibited a storiform and pleomorphic growth pattern with specific myxoid regions (Fig. 1).

Immunohistochemical staining of the MZ-UPS-1 cells and the original tumor from which they were derived, revealed that the cells expressed vimentin and actin and possessed a Ki-67 index of $>20 \%$. The MZ-UPS-2 cells and the cells from their original tumor expressed vimentin and possessed a Ki-67 index of $>20 \%$ (data not shown). The features of the two undifferentiated pleomorphic sarcoma cell lines are revealed in Table II.

Tumor formation following subcutaneous xenotransplantation. Early passages of the two undifferentiated pleomorphic sarcoma cells were used for subcutaneous xenotransplantation into NSG mice. Tumors from the MZ-UPS-1 cells grew $\leq 1.5 \mathrm{~cm}^{3}$ in diameter 4-6 weeks subsequent to a subcutaneous injection of $1 \times 10^{6}$ cells. The histology and type of growth of the tumor was similar to the original human resected tumor (Fig. 1). In the boundary region, the tumor tissue exhibited a solid and predominantly storiform growth pattern with 

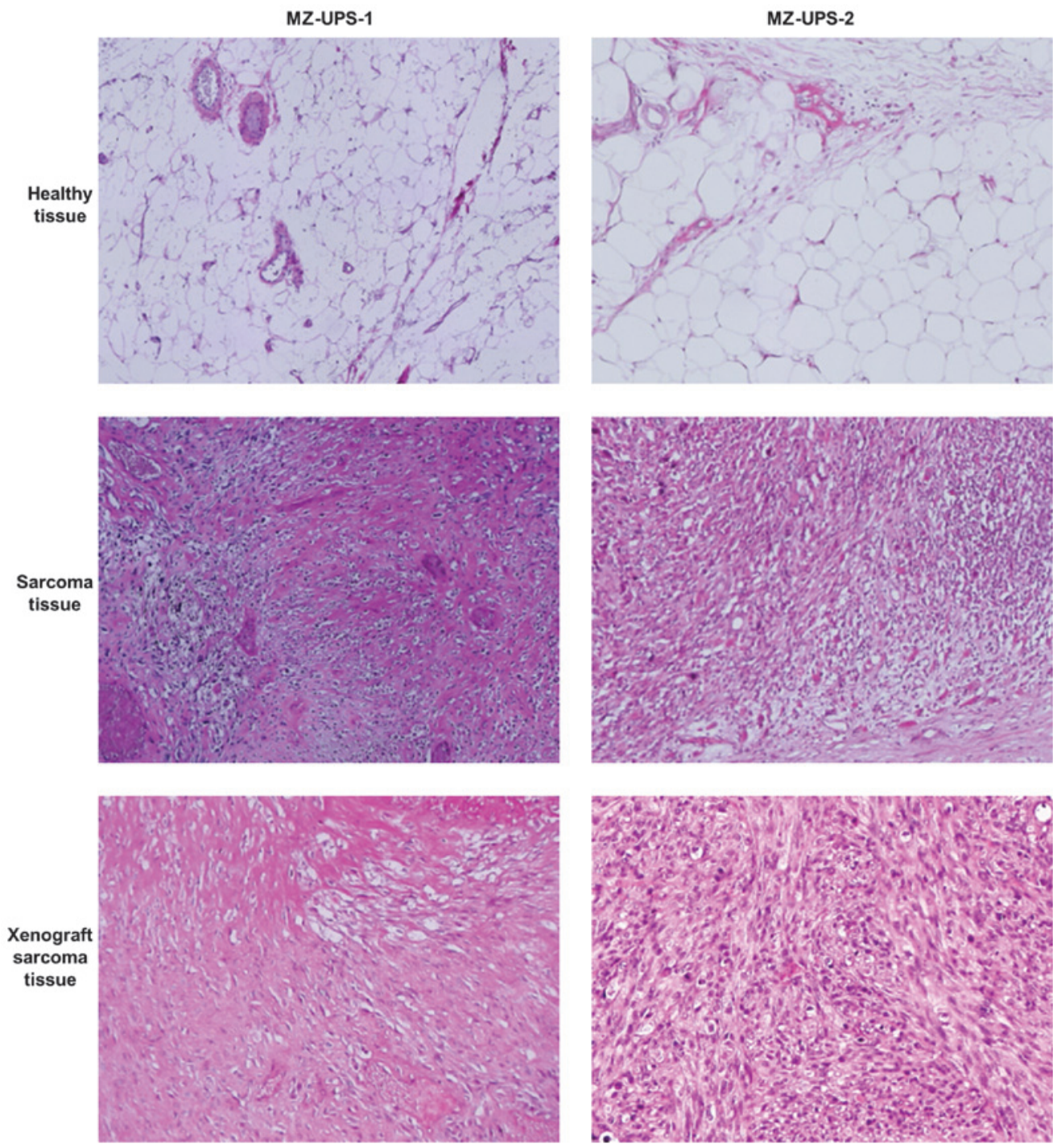

Figure 1. Histology of undifferentiated pleomorphic sarcoma xenograft tumor isolated from NSG mice subjected to xenotransplantation is similar to the original sarcoma tissue resected from human patients. Histological sections of two original human sarcoma tissues (MZ-UPS-1 and MZ-UPS-2), their surrounding healthy tissue and the corresponding xenograft tumors were stained with $\mathrm{H} \& \mathrm{E}$. Xenograft sarcoma tissue was generated by subcutaneous injection of $1 \times 10^{6}$ cultivated MZ-UPS-1 or MZ-UPS-2 cells into the right flank of NSG mice. Following injection, the solid tumor tissue from the xenograft transplant was isolated and fixed 8 weeks later. The isolated tumor tissue was embedded in paraffin, and sections were stained with $\mathrm{H} \& \mathrm{E}$. H\&E, hematoxylin and eosin; NSG, NOD SCID gamma; NOD, non-obese diabetic; SCID, severe combined immunodeficiency.

increased myxoid regions and a fluid-filled chamber. On the surface of the tumor and the surrounding tissue, neovascularization was clearly observed (Fig. 2). Similarly to the in vitro findings, the in vivo tumor formation of MZ-UPS-2 cells was slower, compared with MZ-UPS-1 cells. Histologically, the MZ-UPS-2 xenograft exhibited a predominantly storiform growth pattern; however, no myxoid regions or fluid-filled chambers were observed (Fig. 1), contrarily to the original MZ-UPS-2 tumor tissue. Similarly to the xenograft tumor from the MZ-UPS-1 cells, the MZ-UPS-2 tumor exhibited clear neovascularization on the surface.

Chemotherapeutic treatment of undifferentiated pleomorphic sarcoma cell cultures in vitro. Doxorubicin is commonly used as the first-line treatment for patients with undifferentiated pleomorphic sarcoma. There is no widely accepted second-line treatment. However, novel therapeutic approaches have been recently identified as second-line treatments for patients with recurrent or incurable sarcoma, including HDAC inhibitors and tyrosine kinase inhibitors, such as pazopanib, which has been approved in USA and Europe since 2012 for the treatment of distinct STS subtypes (14-19).

The two patients in the present study were administered doxorubicin; however, this treatment did not prevent tumor progression. Pazopanib was also used in the two patients as a second-line treatment, which resulted in the tumor becoming stable. During pazopanib treatment, symptom relief with increased tumor necrosis was observed in one patient (MZ-UPS-2), and in the other patient a mild tumor regression was observed (data not shown).

Prior to the in vivo experiments, the fourth passages of the two cell cultures, MZ-UPS-1 and MZ-UPS-2, were incubated with various concentrations of doxorubicin, pazopanib and the HDAC inhibitor SAHA, also known as vorinostat (Fig. 3). The anthracycline doxorubicin is a remarkably potent cytostatic drug, and caused the death of the MZ-UPS-1 cells within 
Table II. Two novel undifferentiated pleomorphic sarcoma cell lines.

\begin{tabular}{lll}
\hline Features & \multicolumn{1}{c}{ MZ-UPS-1 } & \multicolumn{1}{c}{ MZ-UPS-2 } \\
\hline Organism & Human & Human \\
Ethnicity & Caucasian & Caucasian \\
Age, years & 47 & 48 \\
Gender & Male & Female \\
Tissue & Mesenchymal & Mesenchymal \\
Morphology & Fibroblastic/myofibroblastic & Fibroblastic/myofibroblastic \\
Cell type & Pleomorphic sarcoma NOS, G3 & Pleomorphic sarcoma NOS, G3 \\
Growth properties & Monolayer & Monolayer \\
Culture medium & DMEM/F12-GlutaMAX ${ }^{\mathrm{TM}}$ Supplement & DMEM/F12-GlutaMAX ${ }^{\mathrm{TM}}$ Supplement \\
Split ratio & with sodium pyruvate and $10 \%$ FCS & with sodium pyruvate and 10\% FCS \\
Medium renewal & $1: 3-1: 5$ every week & $1: 2$ every 2 weeks \\
Tumorigenic & $2-3$ times weekly & $2-3$ times weekly \\
& Yes, in NSG mice & Yes, in NSG mice
\end{tabular}

NOS, not otherwise specified; G3, grade 3; DMEM/F12, Dulbecco's Modified Eagle's Medium: Nutrient Mixture F-12; FCS, fetal calf serum; NSG, NOD SCID gamma; NOD, non-obese diabetic; SCID, severe combined immunodeficiency.
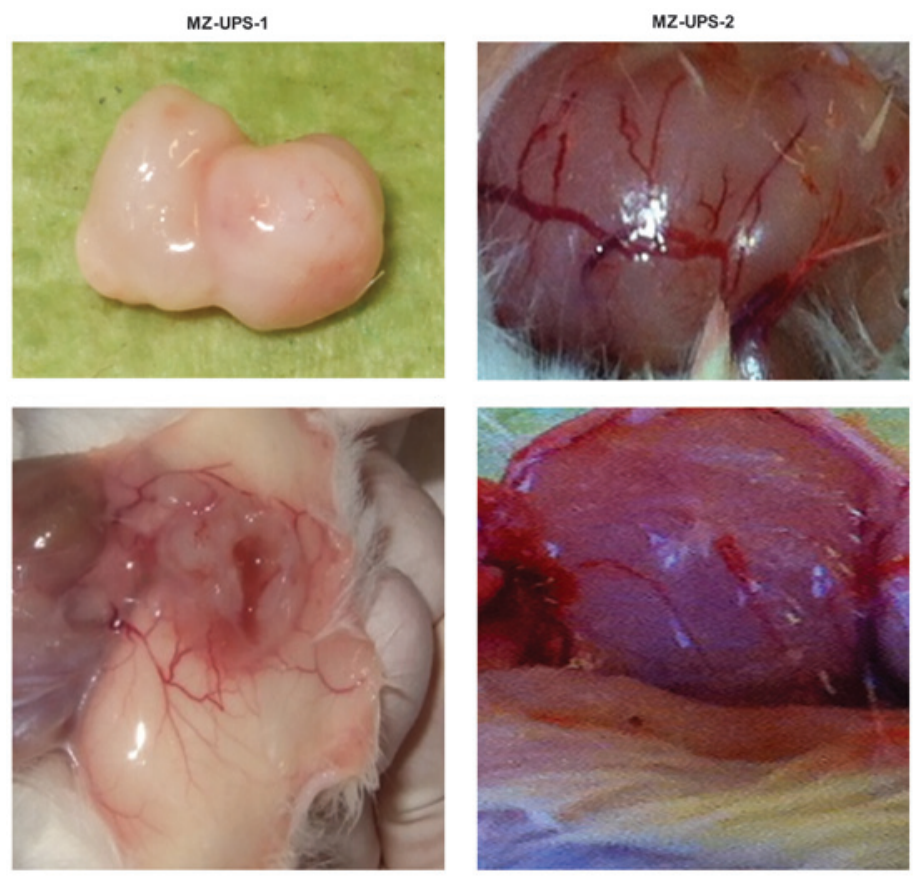

Figure 2. Tumor xenografts observed at 8-12 weeks subsequent to subcutaneous injection of undifferentiated pleomorphic sarcoma cells in NSG mice. Cultivated MZ-UPS-1 or MZ-UPS-2 cells were harvested and washed twice in phosphate-buffered saline. A total of $1 \times 10^{6}$ cells of each culture were injected subcutaneously into the right flank of NSG mice. Solid tumor tissues of $\leq 1.5 \mathrm{~cm}^{3}$ in size were observed in all three pleomorphic sarcomas at 8-12 weeks following injection. NSG, NOD SCID gamma; NOD, non-obese diabetic; SCID, severe combined immunodeficiency.

14 days, even at a concentration of $50 \mathrm{nM}$. The viability of the MZ-UPS-2 cells began to decrease on day 7 following incubation with the highest concentration of doxorubicin tested (100 nM). On day 14, the viability of the MZ-UPS-2 cells was considerably decreased, even at a concentration of $10 \mathrm{nM}$ doxorubicin. The viability of the two cell cultures was decreased on day 4 following incubation in the presence of 5 or $10 \mu \mathrm{M}$ vorinostat. Pazopanib had no inhibitory effect on MZ-UPS-1 cells, and slightly inhibited the growth of MZ-UPS-2 cells. The combination of doxorubicin and pazopanib had no synergistic effect on MZ-UPS-1 cells, and only caused a small synergistic reduction in the viability of MZ-UPS-2 cells.

Chemotherapeutic treatment in vivo. Immunodeficient NSG mice with xenografts of MZ-UPS-1 or MZ-UPS-2 cells were generated in the present study to compare the efficacy of different chemotherapeutic treatments in vivo (which is expected to be comparable to human patient response) with the cytostatic effects demonstrated by these chemotherapeutic drugs in vitro. 
MZ-UPS-1
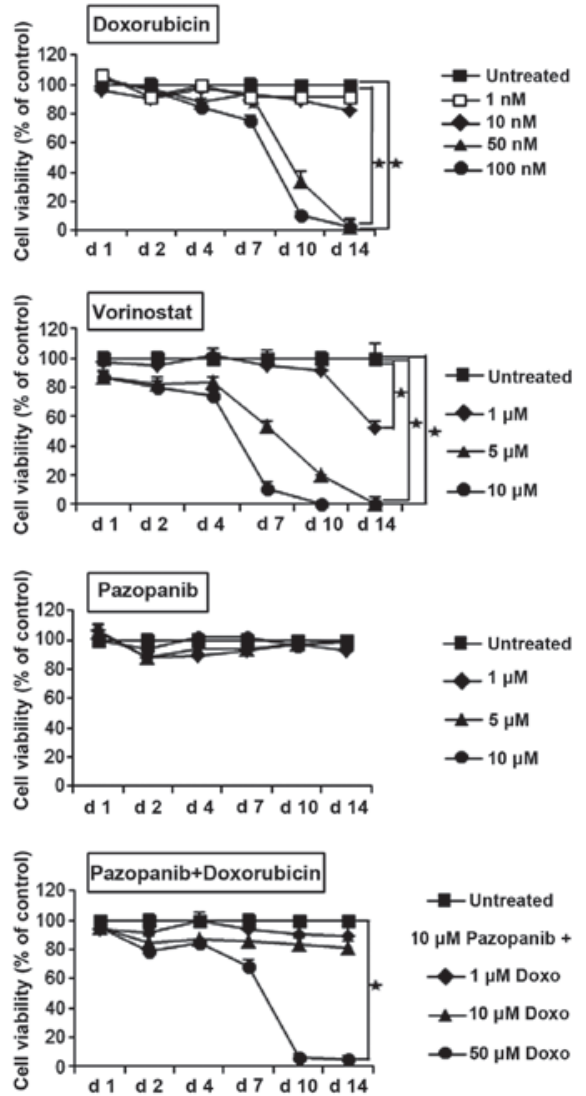

MZ-UPS-2
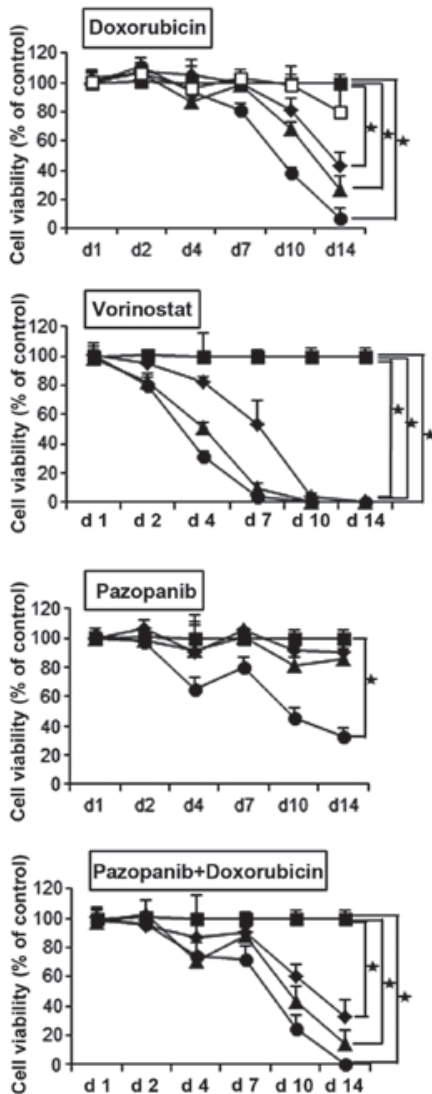

Figure 3. Chemotherapeutic treatment of undifferentiated pleomorphic sarcoma cells in vitro. Cultured cells from two patients with undifferentiated pleomorphic sarcoma, MZ-UPS-1 and MZ-UPS-2, were seeded onto 96-well plates at a concentration of 1x104 cells/100 $\mu 1$ medium/well. Doxorubicin was added on day 0 and 7 , while vorinostat and pazopanib were added daily. For the determination of cell viability, an alamarBlue ${ }^{\mathrm{TM}}$ assay was performed on days 1,2 , 4, 7, 10 and 14. The metabolized substrate was measured photometrically at $570 \mathrm{~nm}$. All experiments were performed in triplicate. Data are presented as the mean \pm standard deviation. "P $\leq 0.01$ vs. untreated cells.

A

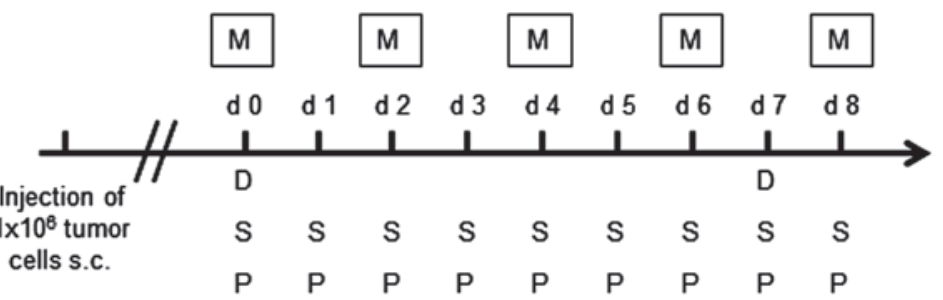

B
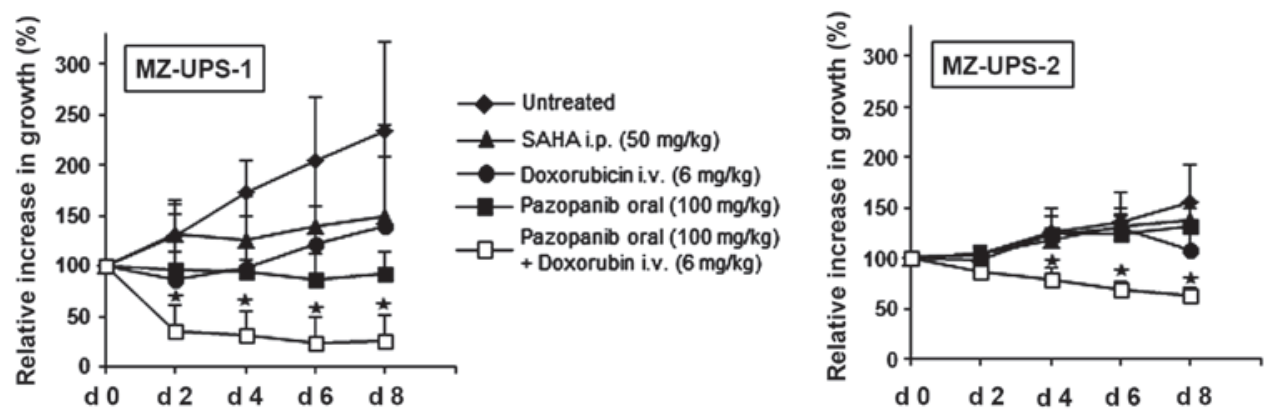

Figure 4. Combined therapy with doxorubicin and pazopanib resulted in a reduction of tumor size in the two xenograft sarcoma mouse models. In vitro cultured MZ-UPS-1 and MZ-UPS-2 cells were washed twice in phosphate-buffered saline, and $1 \times 10^{6}$ cells were injected subcutaneously into NSG mice. Chemotherapeutic treatment was started 6-8 weeks following xenotransplantation. (A) The chemotherapeutic regimen administered to the xenograft sarcoma mouse models was as follows: Doxorubicin was injected intravenously, while suberoylanilide hydroxamic acid and pazopanib were administered intraperitoneally and orally, respectively. (B) The initial tumor size of each mouse was normalized to $100 \%$. Tumor size was measured every 2 days, and compared to the initial size. Relative tumor growth was averaged in each group. Data are presented as the mean \pm standard deviation. $n=5-10 / g r o u p . ~ " P \leq 0.05$ vs. untreated. $M$, measurement; s.c., subcutaneously; D, doxorubicin; S/SAHA, suberoylanilide hydroxamic acid; P, pazopanib; i.p., intraperitoneally; i.v., intravenously. 
Cells from the same passage were isolated and injected into NSG mice. The concentrations of the chemotherapeutic agents used and the frequency of application corresponded to treatment regimens that are normally employed to treat human patients with undifferentiated pleomorphic sarcoma (Fig. 4A), according to the guidelines from the German Society for Hematology and Medical Oncology (www.onkopedia-guidelines.info/en/onkopedia/guidelines). Tumor size from the mouse models was initially measured and normalized to $100 \%$ 6-8 weeks subsequent to the injection of the human undifferentiated pleomorphic sarcoma cells. The tumor size of MZ-UPS-1 mice that were not treated with chemotherapy had more than doubled within 8 days. By contrast, the tumor growth of mice treated with doxorubicin or SAHA was markedly decelerated, and treatment with pazopanib resulted in a stabilization of tumor size. Combined therapy with doxorubicin and pazopanib significantly reduced the tumor to $<50 \%$ of its initial size (Fig. 4B).

The xenograft development of the MZ-UPS-2 cells was slower compared to that of MZ-UPS-1 cells (Fig. 4B), which was comparable to the growth of the MZ-UPS-2 cells in vitro. The tumor size of MZ-UPS-2 mice that were not treated with chemotherapy increased $\sim 0.5$ times in size 8 days subsequent to the initial measurement of the tumor. Following treatment with doxorubicin, SAHA and pazopanib, the pleomorphic tumor xenograft only marginally decreased in size. The combined therapy of doxorubicin and pazopanib led to a significant reduction in the tumor size, and therefore was the most promising therapeutic treatment in vivo. The in vivo results obtained in the present study are markedly different to the in vitro results, where doxorubicin and the HDAC inhibitor SAHA were the most potent chemotherapeutic agents, while pazopanib only marginally influenced tumor cell viability.

\section{Discussion}

Undifferentiated pleomorphic sarcoma is an extremely heterogeneous aggressive subgroup of soft tissue sarcoma (2). Due to the heterogeneity of undifferentiated pleomorphic sarcoma, numerous studies are focused on developing individualized therapeutic strategies for patients, instead of administering a standard chemotherapy to all patients (29). Since there is a limited number of patients affected by each of the undifferentiated pleomorphic sarcoma subgroups, no large clinical trial has been conducted to date to evaluate the efficiency of chemotherapeutic treatment. Therefore, it is crucial to develop preclinical tools that allow the evaluation of individualized therapeutic approaches. Previous studies have demonstrated that there are distinct histopathological differences between the different subtypes of undifferentiated pleomorphic sarcoma, and have revealed a panel of molecular markers that may significantly aid the development of an optimal management regimen for patients with undifferentiated pleomorphic sarcoma $(30,31)$. Consequently, reliable and reproducible preclinical animal models are required, which are similar to the oligoclonal biological diversity observed in human patients, for testing various targeted therapeutic approaches for individual patients.

The present study established two xenograft animal models generated from stable undifferentiated pleomorphic sarcoma cell cultures to investigate the efficacy of chemotherapeutic regimens for the treatment of undifferentiated pleomorphic sarcoma in vivo vs. in vitro. The results of the present study demonstrated that there is a clear discrepancy between the in vitro cell culture and the in vivo xenograft model, which is comparable to a human treatment scenario. The mouse model reflects the local microenvironment of a human tumor, which appears to be crucial to allow a predictive analysis of treatment regimens in addition to monitoring direct cytotoxic effects of drugs (32).

Understanding the various biological sensitivities of the various histological subtypes of undifferentiated pleomorphic sarcoma may lead to the development of individual therapeutic targeted approaches (33).

In contrast to other approaches using solid tumor tissue or silicon chambers to place tumor fragments around the superficial epigastric vessels $(26,27)$, the present study generated stable oligoclonal cell cultures from freshly isolated tumor tissue of two patients with undifferentiated pleomorphic sarcoma, which were similar to the oligoclonal variety exhibited by the original tumors. Additionally, these cultures were subcutaneously injected into immunodeficient mice to establish xenograft animal models. The present study observed that neovascularization was identical between the original tumor and the xenograft tumor. There were no regions of hypoxemia in the xenograft tumor, which is important, as it allows the analysis of anti-angiogenic therapeutic approaches and rules out the possibility of anomalous results that hypoxic conditions may generate during homing and engraftment of the tumor (34).

Furthermore, the present study treated the tumors in vivo and in vitro with the most common or innovative chemotherapeutic agents currently available. Only in the xenograft mouse model the results observed were comparable to the treatment results of the two patients from whom the original tumors were resected. Therefore, tumor derived cell cultures do not reflect the actual treatment condition that is observed in patients. Notably, the combination of doxorubicin and pazopanib significantly reduced the tumor size with an acceptable toxicity level, in terms of weight loss $(<20 \%)$, movement disorder and apathy (data not shown).

In addition, the novel xenograft models allow chemotherapeutic analysis at various time points, which may lead to the identification of molecular mechanisms associated with pleomorphic sarcoma development and progression, and other local tumor-tissue interactions.

In conclusion, there is a discrepancy in tumor growth and cell viability between in vitro and in vivo models concerning chemotherapeutic treatments. The novel and reproducible xenograft animal models generated in the present study have demonstrated that in vivo models are required to test potential chemotherapeutic agents for the treatment of undifferentiated pleomorphic sarcoma, since they provide similar results to those observed in human patients, compared with in vitro models.

\section{Acknowledgements}

The present study was supported by the Internal University Initial Research Founding of the Johannes Gutenberg University of Mainz (Mainz, Germany). 


\section{References}

1. Iwasaki H, Isayama T, Johzaki H and Kikuchi M: Malignant fibrous histiocytoma. Evidence of perivascular mesenchymal cell origin immunocytochemical studies with monoclonal anti-MFH antibodies. Am J Pathol 128: 528-537, 1987.

2. Katenkamp K and Katenkamp D: Soft tissue tumors: New perspectives on classification and diagnosis. Dtsch Arzteb Int 106: 632-636, 2009.

3. Poremba C: Soft tissue sarcomas: The role of histology and molecular pathology for differential diagnosis. Verh Dtsch Ges Pathol 90: 59-72, 2006 (In German).

4. Al-Agha OM and Igbokwe AA: Malignant fibrous histiocytoma: Between the past and the present. Arch Pathol Lab Med 132: 1030-1035, 2008.

5. Matushansky I, Charytonowicz E, Mills J, Siddiqi S, Hricik T and Cordon-Cardo C: MFH classification: Differentiating undifferentiated pleomorphic sarcoma in the 21st Century. Expert Rev Anticancer Ther 9: 1135-1144, 2009.

6. Spira AI and Ettinger DS: The use of chemotherapy in soft-tissue sarcomas. Oncologist 7: 348-359, 2002.

7. Grimer R, Judson I, Peake D and Seddon B: Guidelines for the management of soft tissue sarcomas. Sarcoma 2010: 506182, 2010.

8. Italiano A, Mathoulin-Pelissier S, Cesne AL, Terrier P, Bonvalot S, Collin F, Michels JJ, Blay JY, Coindre JM and Bui B: Trends in survival for patients with metastatic soft-tissue sarcoma. Cancer 117: 1049-1054, 2011.

9. Daigeler A, Klein-Hitpass L, Stricker I, Müller O, Kuhnen C, Chromik AM, Steinstraesser L, Goertz O, Steinau HU and Lehnhardt M: Malignant fibrous histiocytoma - pleomorphic sarcoma, NOS gene expression, histology, and clinical course. A pilot study. Langenbecks Arch Surg 395: 261-275, 2010.

10. Fletcher CDM, Unni KK and Mertens F (eds): World Health Organisation Classification of Tumours. Pathology and Genetics of Tumours of Soft Tissue and Bone. IARC Press, Lyon, 2002.

11. Casali PG and Blay JY; ESMO/CONTICANET/EUROBONET Consensus Panel of experts: Soft tissue sarcomas: ESMO Clinica Practice Guidelines for diagnosis, treatment and follow-up. Ann Oncol 21 (Suppl 5): v198-v203, 2010.

12. Italiano A, Le Cesne A, Mendiboure J, Blay JY, Piperno-Neumann S, Chevreau C, Delcambre C, Penel N, Terrier P, Ranchere-Vince D, et al: Prognostic factors and impact of adjuvant treatments on local and metastatic relapse of soft-tissue sarcoma patients in the competing risks setting. Cancer 120: 3361-3369, 2014

13. Leahy M, Garcia Del Muro X, Reichardt P, Judson I, Staddon A, Verweij J, Baffoe-Bonnie A, Jönsson L, Musayev A, Justo N, et al SABINE Investigators: Chemotherapy treatment patterns and clinical outcomes in patients with metastatic soft tissue sarcoma. The SArcoma treatment and Burden of Illness in North America and Europe (SABINE) study. Ann Oncol 23: 2763-2770, 2012.

14. van der Graaf WT, Blay JY, Chawla SP, Kim DW, Bui-Nguyen B, Casali PG, Schöffski P, Aglietta M, Staddon AP, Beppu Y, et al; EORTC Soft Tissue and Bone Sarcoma Group; PALETTE study group: Pazopanib for metastatic soft-tissue sarcoma (PALETTE) A randomised, double-blind, placebo-controlled phase 3 trial. Lancet 379: 1879-1886, 2012

15. Ray-Coquard I and Thomas D: Targeted therapies: Pazopanib for soft-tissue sarcoma: A PALETTE of data emerges. Nat Rev Clin Oncol 9: 431-432, 2012.

16. Nguyen DT and Shayahi S: Pazopanib: Approval for soft-tissue sarcoma. J Adv Pract Oncol 4: 53-57, 2013.

17. Ranieri G, Mammì M, Donato Di Paola E, Russo E, Gallelli L, Citraro R, Gadaleta CD, Marech I, Ammendola M and De Sarro G: Pazopanib a tyrosine kinase inhibitor with strong anti-angiogenetic activity: A new treatment for metastatic soft tissue sarcoma. Crit Rev Oncol Hematol 89: 322-329, 2014.
18. Tavallai S, Hamed HA, Grant S, Poklepovic A and Dent P: Pazopanib and HDAC inhibitors interact to kill sarcoma cells. Cancer Biol Ther 15: 578-585, 2014.

19. Schöffski P, Cornillie J, Wozniak A, Li H and Hompes D: Soft tissue sarcoma: An update on systemic treatment options for patients with advanced disease. Oncol Res Treat 37: 355-362, 2014.

20. Nakatani T, Marui T, Yamamoto T, Kurosaka M, Akisue T and Matsumoto K: Establishment and characterization of cell line TNMY1 derived from human malignant fibrous histiocytoma. Pathol Int 51: 595-602, 2001.

21. Krause AK, Hinrichs SH, Orndal C, DeBoer J, Neff JR and Bridge JA: Characterization of a human my xoid malignant fibrous histiocytoma cell line, OH931. Cancer Genet Cytogenet 94: 138-143, 1997.

22. Mairal A, Chibon F, Rousselet A, Couturier J, Terrier P and Aurias A: Establishment of a human malignant fibrous histiocytoma cell line, COMA. Characterization by conventional cytogenetics, comparative genomic hybridization, and multiplex fluorescence In situ hybridization. Cancer Genet Cytogenet 121: 117-123, 2000.

23. Hakozaki M, Hojo H, Sato M, Tajino T, Yamada H, Kikuchi S and Abe M: Establishment and characterization of a new cell line, FPS-1, derived from human undifferentiated pleomorphic sarcoma, overexpressing epidermal growth factor receptor and cyclooxygenase-2. Anticancer Res 26 (5A): 3393-3401, 2006.

24. Nishio J, Iwasaki H, Nabeshima K, Ishiguro M, Isayama T and Naito M: Establishment of a new human pleomorphic malignant fibrous histiocy toma cell line, FU-MFH-2: Molecular cytogenetic characterization by multicolor fluorescence in situ hybridization and comparative genomic hybridization. J Exp Clin Cancer Res 29: 153, 2010.

25. Steinstraesser L, Jacobsen F, Schubert C, Gevers K, Stricker I, Steinau HU and Al-Benna S: Establishment of a primary human sarcoma model in athymic nude mice. Hum Cell 23: 50-57, 2010.

26. Tilkorn DJ, Daigeler A, Hauser J, Ring A, Stricker I, Schmitz I, Steinstraesser L, Steinau HU and Al-Benna S: A novel xenograft model with intrinsic vascularisation for growing undifferentiated pleomorphic sarcoma NOS in mice. J Cancer Res Clin Oncol 138: 877-884, 2012

27. Tilkorn DJ, Stricker I, Hauser J, Ring A, Schmitz I, Steinstraesser L, Steinau HU, Daigeler A and Al-Benna S: Experimental murine model of primary high grade undifferentiated pleomorphic sarcoma not otherwise specified. In Vivo 26: 559-563, 2012.

28. Fletcher CDM, Bridge JA, Hogendoorn P, Mertens F (eds): World Health Organization Classification of Tumours of Soft Tissue and Bone. 4th edition. Volume 5. IARC, Lyon, 2013.

29. Radaelli S, Stacchiotti S, Casali PG and Gronchi A: Emerging therapies for adult soft tissue sarcoma. Expert Rev Anticancer Ther 14: 689-704, 2014.

30. Reichardt P: Soft tissue sarcomas, a look into the future: Different treatments for different subtypes. Future Oncol 10 (Suppl 8): s19-s27, 2014

31. Villacis RA, Silveira SM, Barros-Filho MC, Marchi FA, Domingues MA, Scapulatempo-Neto C, Aguiar S Jr, Lopes A, Cunha IW and Rogatto SR: Gene expression profiling in leiomyosarcomas and undifferentiated pleomorphic sarcomas: SRC as a new diagnostic marker. PLoS One 9: e102281, 2014.

32. Villacis RA, Silveira SM, Barros-Filho MC, Marchi FA, Domingues MA, Scapulatempo-Neto C, Aguiar S Jr, Lopes A, Cunha IW and Rogatto SR: Gene expression profiling in leiomyosarcomas and undifferentiated pleomorphic sarcomas: SRC as a new diagnostic marker. PLoS One 9: e102281, 2014.

33. Versleijen-Jonkers YM, Vlenterie M, van de Luijtgaarden AC and van der Graaf WT: Anti-angiogenic therapy, a new player in the field of sarcoma treatment. Crit Rev Oncol Hematol 91: $172-185,2014$.

34. Tentler JJ, Tan AC, Weekes CD, Jimeno A, Leong S, Pitts TM, Arcaroli JJ, Messersmith WA and Eckhardt SG: Patient-derived tumour xenografts as models for oncology drug development. Nat Rev Clin Oncol 9: 338-350, 2012. 